\title{
Methacholine Dose-Response Slopes from Maximal Bronchial Challenge Tests in Asthmatic Children: Methodological Aspects
}

T. Gamboa, ${ }^{1}$ N. Neuparth, ${ }^{1}$ I. Ribeiro da Silva,,${ }^{1}$ J. E. Rosado Pinto, ${ }^{2}$ and A. B. Rendas ${ }^{1}$

${ }^{1}$ Department of Pathophysiology, Faculty of Medical Sciences, UNL and ${ }^{2}$ Immunoallergology, Hospital Dona Estefânia, Lisbon, Portugal

Abstract. To determine whether the slope of a maximal bronchial challenge test (in which $\mathrm{FEV}_{1}$ falls by over 50\%) could be extrapolated from a standard bronchial challenge test (in which $\mathrm{FEV}_{1}$ falls up to 20\%), 14 asthmatic children performed a single maximal bronchial challenge test with methacholine (dose range: 0.097$30.08 \mu \mathrm{mol})$ by the dosimeter method. Maximal dose-response curves were included according to the following criteria: (1) at least one more dose beyond a $\Delta \mathrm{FEV}_{1} \geqslant 20 \%$; and (2) a $\mathrm{MFEV}_{1} \geqslant 50 \%$. $\mathrm{PD}_{20} \mathrm{FEV}_{1}$ was calculated, and the slopes of the early part of the dose-response curve (standard dose-response slopes) and of the entire curve (maximal dose-response slopes) were calculated by two methods: the two-point slope (DRR) and the least squares method (LSS) in \% $\Delta \mathrm{FEV}_{1} \times \mu \mathrm{mol}^{-1}$. Maximal dose-response slopes were compared with the corresponding standard dose-response slopes by a paired Student's $t$ test after logarithmic transformation of the data; the goodness of fit of the LSS was also determined. Maximal dose-response slopes were significantly different $(p<0.0001)$ from those calculated on the early part of the curve: $\operatorname{DRR}_{20 \%}\left(91.2 \pm 2.7 \Delta \mathrm{FEV}_{1} \% \cdot \mu \mathrm{mol}^{-1}\right)$ was 2.88 times higher than $\mathrm{DRR}_{50 \%}\left(31.6 \pm 3.4 \Delta \mathrm{FEV}_{1} \% \cdot \mu \mathrm{mol}^{-1}\right)$, and the $\mathrm{LSS}_{20 \%}$ $\left(89.1 \pm 2.8 \% \Delta \mathrm{FEV}_{1} \cdot \mu \mathrm{mol}^{-1}\right)$ was 3.10 times higher than $\mathrm{LSS}_{50 \%}(28.8 \pm 1.5 \%$ $\left.\Delta \mathrm{FEV}_{1} \cdot \mu \mathrm{mol}^{-1}\right)$. The goodness of fit of $\mathrm{LSS}_{50 \%}$ was significant in all cases, whereas $\mathrm{LSS}_{20 \%}$ failed to be significant in one. These results suggest that maximal dose-response slopes cannot be predicted from the data of standard bronchial challenge tests.

Key words: Bronchial hyperresponsiveness-Methacholine-Maximal doseresponse curves-Least squares slope-Two-point slope. 


\section{Introduction}

Bronchial hypersensitivity to inhaled pharmacologic agents such as methacholine and histamine is one of the hallmarks of asthma. This means that in asthmatic patients the provocative dose (PD) or concentration (PC) that induces a $20 \%$ fall in the $\mathrm{FEV}_{1}$ is lower than in normal subjects. Although PD and PC are the indexes used most commonly in bronchial challenge tests, giving information about the degree of bronchial sensitivity to the inhaled agent $[6,11,32]$, it is not possible to describe the severity of airway narrowing, based on these parameters alone. This means that asthmatics with similar PDs may reach different degrees of airway obstruction if further doses are inhaled. Thus, the degree of airway sensitivity to a pharmacologic agent provides only a partial information about the severity of bronchial hyperresponsiveness. Furthermore, the effects of antiasthma drugs such as inhaled corticosteroids are frequently monitored by bronchial challenge tests with methacholine and histamine, and PD and PC are the parameters chosen to express the results; however, these changes are often very small $[5,33]$.

In 1993, the European Respiratory Society (ERS) [29] published recommendations for bronchial challenge tests, indicating that complete information from a bronchial challenge test with pharmacologic agents can only be obtained from analysis of the shape of the dose-response curve (DRC). It is clearly stated in this report that, although PD or PC indicates the position of the DRC, the slope and the degree of maximal obstruction provide relevant information about the level and potential severity of bronchial response [9, 27, 29]. Woolcock et al. [34] were the first to demonstrate that the shape of DRC in asthmatics was different from that of normals, i.e. many asthmatics show a higher slope and a higher maximal response or plateau when exposed to high doses of methacholine, histamine, or other inhaled bronchoconstrictors $[2,15,21$, 22, 24]. However, although the ERS report [29] considered that maximal DRCs, mandatory for the study of maximal response, can be safely recorded up to a $50 \%$ decline in $\mathrm{FEV}_{1}$, provided the patients have normal baseline lung function, it is also recommended that this procedure should be limited to research studies. Thus, in a clinical setting, most of the information that can be obtained from bronchial challenge tests with pharmacologic agents comes from the analysis of the early part of the DRC.

Moderate and severe asthmatics are characterized by excessive bronchial narrowing. Such patients do not usually exhibit the stable response (plateau) common in normal subjects or patients with mild asthma. Consequently, in the absence of a plateau, the dose-response slope (DRS) is the only index that may be used to describe the shape of DRC and hence the severity of bronchial response. Although its main application is for epidemiologic studies, the DRS has also been used in clinical [3, 25] and pathophysiologic research [7,31], which often includes the study of patients with moderate to severe asthma. However, there is still some controversy concerning the methods used to calculate and analyze the DRS. First, different groups use different methods to estimate the slopes, and there is no one single model that provides the best fit to all DRCs of asthmatics. Second, although it is known that a linear relationship can be inaccurate in describing the shape of DRCs, the more frequently used methods are the simple linear regression $[1,10,24,34]$ using the least-squares method and the dose-response ratio, or two-point slope, first described by O'Connor et al. [17]. Third, 
Table 1. Subject characteristics and $\mathrm{PD}_{20} \mathrm{FEV}_{1}$ and $\mathrm{MFEV}_{1}$

\begin{tabular}{lllllll}
\hline $\begin{array}{l}\text { Subject } \\
\text { no. }\end{array}$ & Sex & $\begin{array}{l}\text { Age } \\
\text { (years })\end{array}$ & $\begin{array}{l}\text { Height } \\
(\mathrm{cm})\end{array}$ & $\begin{array}{l}\text { Baseline } \mathrm{FEV}_{1} \\
(\% \text { predicted })\end{array}$ & $\begin{array}{l}\mathrm{PD}_{20} \mathrm{FEV}_{1} \\
(\mu \mathrm{mol})\end{array}$ & $\begin{array}{l}\mathrm{MFEV}_{1}{ }^{a} \\
(\% \text { postsaline })\end{array}$ \\
\hline 1 & $\mathrm{M}$ & 10 & 145 & 81 & 0.29 & -54 \\
2 & $\mathrm{M}$ & 13 & 153 & 124 & 0.16 & -54 \\
3 & $\mathrm{~F}$ & 17 & 161 & 108 & 0.39 & -50 \\
4 & $\mathrm{M}$ & 9 & 134 & 97 & 3.76 & -46 \\
5 & $\mathrm{~F}$ & 13 & 150 & 72 & 0.03 & -50 \\
6 & $\mathrm{M}$ & 14 & 155 & 108 & 0.09 & -58 \\
7 & $\mathrm{~F}$ & 15 & 169 & 82 & 0.86 & -50 \\
8 & $\mathrm{~F}$ & 14 & 163 & 114 & 0.40 & -50 \\
9 & $\mathrm{~F}$ & 13 & 147 & 74 & 0.11 & -62 \\
10 & $\mathrm{M}$ & 17 & 178 & 76 & 0.17 & -50 \\
11 & $\mathrm{M}$ & 12 & 151 & 105 & 0.16 & -52 \\
12 & $\mathrm{M}$ & 9 & 132 & 102 & 0.25 & -57 \\
13 & $\mathrm{M}$ & 12 & 139 & 108 & 3.38 & $-30^{b}$ \\
14 & $\mathrm{M}$ & 9 & 143 & 103 & 0.36 & -50 \\
& & & & & & \\
Mean \pm S.D. & 5F/9M & $12.6 \pm 2.7$ & $151.4 \pm 13.1$ & $96.7 \pm 16.5$ & $0.74 \pm 1.22$ & $50.9 \pm 7.3$ \\
\hline
\end{tabular}

${ }^{a} \mathrm{MFEV}_{1}$, maximal reduction of $\mathrm{FEV}_{1}$ postchallenge

${ }^{b}$ DRC with plateau average of two points

the slope is more often determined on standard DRCs (SDRCs) [2, 3, 15, 17, 21, 22, 34] than in maximal DRCs (MDRCs) even in clinical settings [7, 14, 31, 34], and it is not yet clear which portion of the DRC is most important. Although different mathematical models $[2,9,10,17,28,31]$ have been used to describe and/or predict the shape of individual DRCs, we are not aware of any studies determining whether the linear slope of the DRC in asthmatic patients changes significantly when high doses of methacholine are inhaled.

The aim of this study was to determine whether the slope of a maximal bronchial challenge test (in which $\mathrm{FEV}_{1}$ falls by more than 50\%) could be extrapolated from a standard bronchial challenge test (in which $\mathrm{FEV}_{1}$ falls up to 20\%). This may be relevant when deciding whether standard or maximal bronchial challenge tests should be chosen if the slope is used to monitor the degree of bronchial hyperresponsiveness in clinical research protocols.

\section{Materials and Methods}

\section{Study Subjects}

The data on the subjects included in the present study are summarized in Table 1. Fourteen asthmatic children (five females and nine males), aged 9-17 years, whose parents gave their consent after an oral explanation about the protocol, were included. According to their clinical symptoms and/or the daily medication required, ten patients were classified as having moderate to severe asthma, and four were labeled as mild persistent [16]. At the time of the test, all children were clinically stable and free from upper respiratory infections for at least 6 weeks. Baseline $\mathrm{FEV}_{1}$, determined as described below, was $>70 \%$ of the predicted value (Table 1). 
Inhaled bronchodilators and oral theophylline compounds, when used, were withheld at least 8 and $12 \mathrm{hr}$, respectively, before the test [29]. Five patients were taking daily inhaled corticosteroids, two cromolyn sodium and two ketotifen, and this treatment was maintained.

\section{Study Design}

All children performed a single bronchial challenge test with methacholine. Double doses of methacholine were administered with a dosimeter up to a $50 \%$ reduction compared with the postsaline $\mathrm{FEV}_{1}$ or until a plateau response was reached. To determine if the degree of airway obstruction to high doses of methacholine could be predicted from a standard bronchial challenge test, the slopes of MDRC were compared with those obtained on the early part of the same dose-response curve (SDRC).

The slopes were calculated by two methods: the two-point slope or dose-response ratio (DRR) as described by O'Connor et al. [17], and the least squares method (LSS).

The study design was approved by the Scientific Council of the Faculty of Medical Sciences, as part of a master's thesis.

\section{Methacholine Challenge Test}

Forced expiratory maneuvers were measured using a pneumatocograph (model Compact, Vitalograph, Buckingham, England). Baseline and postchallenge $\mathrm{FEV}_{1}$ were recorded as the maximum of three consecutive measurements that agreed with each other within $5 \%$.

The methacholine challenge test was performed using a modified dosimeter method [8], following the European Respiratory Society recommendations [29]. Aerosols were delivered from a dosimeter (model MB3 Mefar, Brescia, Italy) at 20 p.s.i., with an airflow of 8 liters/min and with a nebulizer output of 30.8 $\mu \mathrm{l} /$ five puffs of $0.6 \mathrm{~s}$ each. After inhalation of saline, and if the $\mathrm{FEV}_{1}$ postsaline fell no more than $10 \%$ from baseline value, double cumulative doses of methacholine chloride ranging from 0.097 to $30.08 \mu \mathrm{mol}$ were administered. The aerosols were inhaled by fast inspiratory maneuvers from functional residual capacity (FRC) to total lung capacity (TLC), without breath holding. All subjects wore a nose clip during aerosol inhalation and spirometry. Measurements of $\mathrm{FEV}_{1}$ postchallenge were performed $90 \mathrm{~s}$ after saline and after each dose of methacholine. The test was interrupted if one of the following occurred: (1) a $\mathrm{FEV}_{1}$ decline $\geqslant 50 \%$; (2) a plateau was reached, i.e., when the last two response values showed a variation of less than 5\% between each other; (3) at the subject's request due to respiratory symptoms; or (4) after the last dose (30.08 $\mu \mathrm{mol})$ had been given.

Airway sensitivity was expressed as the $\mathrm{PD}_{20} \mathrm{FEV}_{1}$, obtained from the semilogarithmic DRC by linear interpolation of the last two points, according to the formula recommended by ERS [29].

Maximal bronchoconstrictor response $\left(\mathrm{MFEV}_{1}\right)$ was expressed as the maximal percentage fall in $\mathrm{FEV}_{1}$ $[29,34]$; otherwise, whenever the response reached a plateau the data points on the plateau were averaged [30-32].

\section{Data Analysis}

The curves were included in the statistical analysis according to the following criteria: (1) at least one more dose beyond a $\mathrm{FEV}_{1}$ reduction $\geqslant 20 \%$, and (2) a $\mathrm{MFEV}_{1} \geqslant 50 \%$ without plateau.

\section{Least Squares Slope}

As shown in Figure 1, methacholine DRSs were determined by the LSS in two ways: (1) with all data points of MDRCs $\left(\mathrm{LSS}_{50 \%}\right)$ from the first to the last cumulative dose or to the first cumulative dose at which a plateau response $\geqslant 50 \%$ was reached [7]; and (2) from the first dose of methacholine to the cumulative dose 


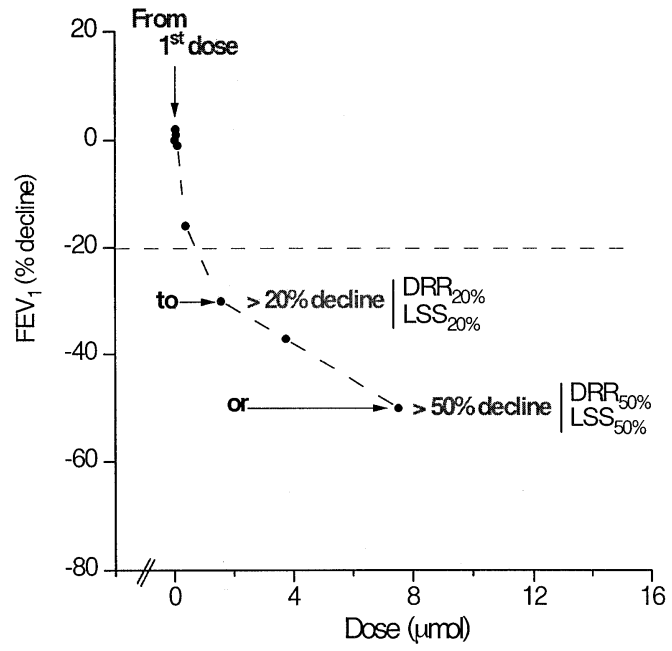

Fig. 1. Data points used to calculate the slopes from standard and maximal DRCs.

at which $\mathrm{FEV}_{1}$ reduction was $\geqslant 20 \%$ from postsaline value $\left(\mathrm{LSS}_{20 \%}\right)$ [10]. Only the LSS with at least three data points were included in the analysis.

\section{Two-point Slope (DRR)}

The slope of the straight line between the origin and the final cumulative dose was also determined by the simplified formula (Equation 1), the two-point slope or DRR proposed by O'Connor et al. [17]:

$$
\mathrm{DRR}=\Delta \mathrm{FEV}_{1}(*) / \operatorname{dose}^{2} \Delta \mathrm{FEV}_{1} \times \mu \mathrm{mol}^{-1}
$$

(*) $\Delta \mathrm{FEV}_{1}(\%)=\left[\left(\right.\right.$ postsaline $\mathrm{FEV}_{1}-$ last $\left.\mathrm{FEV}_{1}\right) /$ postsaline $\left.\mathrm{FEV}_{1}\right] \times 100$

\section{Statistical Methods}

The goodness of fit of LSS was expressed by the coefficient of determination $r^{2}$, and the linear regression was considered significant if $p<0.05$. The median of the number of points included in the calculation of $\mathrm{LSS}_{20 \%}$ and $\mathrm{LSS}_{50 \%}$ was also determined.

A Kolmogorov-Smirnov one-sample test was used to compare the distributions of the LSS and DRR with the normal distribution, and the Levene test was applied to test the homogeneity of variances. After logarithmic transformation ( $\log 10)$, the distributions of the maximal and standard DRS in this group were not significantly different from the normal, and the variances between groups $\left(\mathrm{DRR}_{20 \%}\right.$ and $\mathrm{DRR}_{50 \%}$; $\mathrm{LSS}_{20 \%}$ and $\mathrm{LSS}_{50 \%}$ ) were homogeneous.

Paired Student's $t$ tests were performed to compare the slopes of the SDRC with those determined using all data points in the MDRC: (1) $\mathrm{LSS}_{20 \%}$ with $\mathrm{LSS}_{50 \%}$ and (2) the standard dose-response ratio $\left(\mathrm{DRR}_{20 \%}\right)$ with maximal dose-response ratio $\left(\mathrm{DRR}_{50 \%}\right)$. The differences were considered statistically significant if $p<$ 0.05 .

After statistical analysis, the results were recalculated into the standard form and the results expressed as geometric mean \pm S.D.

\section{Results}

From Table 1 it is apparent that all children tested had hypersensitivity to methacholine (range $\mathrm{PD}_{20}, 0.03-3.76 \mu \mathrm{mol}$ ), and 12 reached a $\mathrm{FEV}_{1}$ decline $\geqslant 50 \%$. Two subjects 


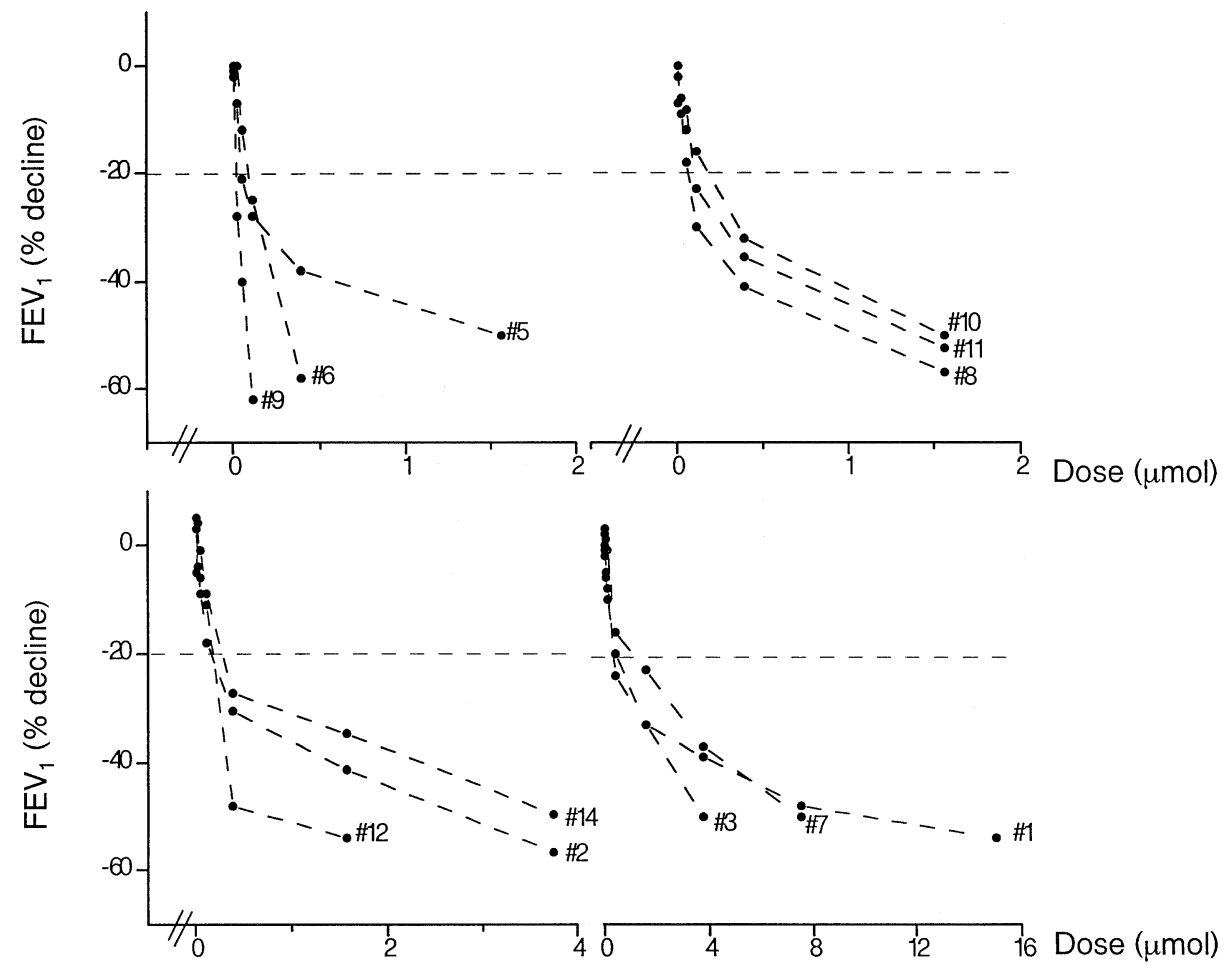

Fig. 2. Individual DRCs from the 12 subjects.

were excluded. In subject 4 the test was stopped because of breathlessness, and subject 13 reached a plateau at $30 \%$. Therefore, it was possible to compare the slopes in 12 subjects, whose individual DRC are shown in Figure 2.

In Table 2 it is shown that the coefficients of determination of $\mathrm{LSS}_{20 \%}$ and $\mathrm{LSS}_{50 \%}$ were all above $0.80 . \mathrm{LSS}_{50 \%}$ reached statistical significance $(p<0.05)$ in all cases, whereas $\mathrm{LSS}_{20 \%}$ failed to be significant in just one case, with only three points included in the regression. Since there were no zero or negative slopes, there was no need to add a constant prior to the logarithmic transformation.

\section{Comparison of MDRS with SDRS}

The individual values of MDRS and SDRS calculated by both methods are represented in Figure 3. MDRSs were significantly different $(p<0.0001)$ from those determined in the early part of the same DRC. The slopes calculated on the early part of the DRC were higher than MDRS, both with the DRR [DRR $20 \%\left(91.2 \pm 2.7 \% \Delta \mathrm{FEV}_{1} \times \mu \mathrm{mol}^{-1}\right)$ was 2.88 times higher than $\left.\operatorname{DRR}_{50 \%}\left(31.6 \pm 3.4 \Delta \mathrm{FEV}_{1} \% \times \mu \mathrm{mol}^{-1}\right)\right]$ and with the LSS $\left[\operatorname{LSS}_{20 \%}\left(89.1 \pm 2.8 \% \Delta \mathrm{FEV}_{1} \times \mu \mathrm{mol}^{-1}\right)\right.$ was 3.1 times higher than $\operatorname{LSS}_{50 \%}(28.8 \pm$ $\left.\left.1.5 \% \Delta \mathrm{FEV}_{1} \times \mu \mathrm{mol}^{-1}\right)\right]$. 
Table 2. Goodness of the fit of $\operatorname{LSS}_{20 \%}$ and $\operatorname{LSS}_{50 \%}(n=12)$

\begin{tabular}{llllll}
\hline & $\begin{array}{l}\text { No. points } \\
(\text { min }- \text { max })\end{array}$ & $\begin{array}{l}\text { No. points } \\
\text { (median) }\end{array}$ & $r^{2 b}$ & $\begin{array}{l}\text { No. slopes with } \\
r^{2} \geqslant 0.95\end{array}$ & $\begin{array}{l}\text { No. slopes with } \\
p>0.05^{c}\end{array}$ \\
\hline $\mathrm{LSS}_{20 \%}$ & $3-8$ & 4.5 & $0.83-0.99$ & 10 & 11 \\
$\mathrm{LSS}_{50 \%}$ & $4-9$ & 6 & $0.88-0.99$ & 11 & 12 \\
\hline
\end{tabular}

${ }^{a}$ No. points, number of points included; min, minimum; max, maximum

${ }^{b} r^{2}$, coefficients of determination of linear regression

${ }^{c} p$, statistical significance of linear regression
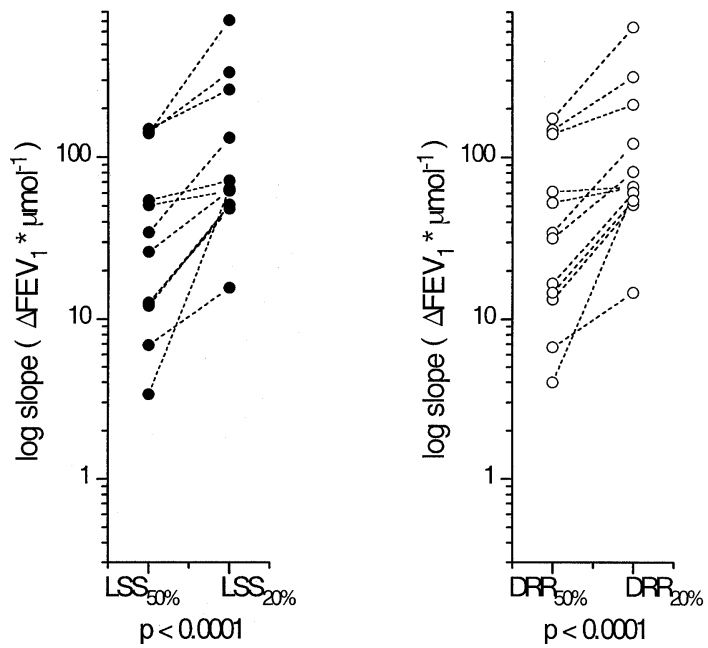

Fig. 3. Comparison between individual slopes calculated by the two methods (LSS or DRR), using the standard part of the curve (20\%) and the entire curve (50\%). Each point $(\checkmark$ and $\bigcirc)$ represents the individual value of the slope $(n=12)$.

\section{Discussion}

The purpose of the present study was to determine if MDRS could be extrapolated from the slope of a standard bronchial challenge test, which is still the recommended procedure to evaluate the degree of bronchial hyperresponsiveness in clinical and epidemiologic protocols. From the analysis of this group of asthmatic children it was clear that the degree of airway narrowing induced by a maximal bronchial challenge test with methacholine could not be predicted from the slopes determined in the early part of the same MDRC. The following differences between SDRS and MDRS were observed with both methods of calculation of the slopes. First, both mean $\mathrm{DRR}_{20 \%}$ and $\mathrm{LSS}_{20 \%}$ (standard slopes) overestimated almost by three times the slopes measured on the same DRC using all data points up to a fall of $50 \%$ or more from baseline (maximal slopes). Second, although these methods of assessing the DRC are oversimplified because both assume that the dose-response relationship is linear, the analysis of the goodness of fit 
of LSS in this group of asthmatics showed that the coefficients of determination $\left(r^{2}\right)$ of $\mathrm{LSS}_{20 \%}$ and $\mathrm{LSS}_{50 \%}$ were all above 0.80 . The $\mathrm{LSS}_{50 \%}$ reached statistical significance in all cases, whereas $\mathrm{LSS}_{20 \%}$ failed to be significant in only one. The low grade of significance found in this case was probably due to an insufficient number of data points included in the calculation (three points). Thus, although the relationships between increasing doses of methacholine and the severity of airway response may have changed during the test and the methods of calculating the slopes are still open to debate $[1,2,10,12,23,26,28]$, these results suggested that a linear model can be used to calculate this index of bronchial hyperresponsiveness in asthmatic patients. In fact, the linear model is the most frequently used not only in epidemiologic $[1,4,7,18,24]$ but also in research protocols $[3,13,15,19,34]$. The mean values of $\mathrm{DRR}_{20 \%}$ measured in our group of patients were well above the cutoff levels between normals and asthmatics, established with the same method of calculation by Peat et al. [21] and by Backer [4] in epidemiologic studies in children. However, these values were obtained from standard bronchial challenge tests with histamine, and it is still not known if the slopes of DRC to histamine and methacholine in children are comparable [20, 27]. Concerning the least squares method, the $\mathrm{LSS}_{20 \%}$ values found in the present study were similar or higher than the mean values calculated by Chinn et al. [10] in adult subjects with symptoms of asthma, and the $\mathrm{LSS}_{50 \%}$ were within the same range as those measured in clinical trials of adult asthmatic subjects $[3,25]$.

To our knowledge, no other studies have compared SDRS with MDRS using the present methods. This may be because most of the maximal bronchial challenge tests were performed in mild asthmatics who usually exhibited a reproducible plateau response, which is generally accepted to describe the shape of DRC. Beyond its usefulness in epidemiologic studies, thus avoiding the censoring of data, other groups have used the slope together with PD and/or plateau to monitor the effects of inhaled antiinflammatory drugs $[3,25]$ or natural antigenic exposure in bronchial reactivity of asthmatics and subjects with allergic rhinitis [7]. In this kind of research, where moderate and severe asthmatics might be included, the slope may be the only additional parameter available to describe the shape of DRC. Since the slope is measured on the steepest part of the DRC, the differences between SDRS and MDRS are expected to be even higher in more severe asthmatics without plateau than in normal subjects or patients in whom a stable response is obtained.

Our results suggest that MDRS is the closest measurement of the potential severity of airway hyperresponsiveness which can be obtained from an artificial situation such as a bronchial challenge test when a plateau response is absent.

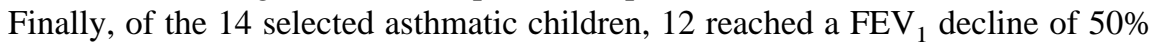
or more from postsaline and exhibited excessive airway narrowing without plateau, and so the analysis of the shape of DRC could only be provided by $\mathrm{PD}_{20} \mathrm{FEV}_{1}$ and slope. These results were obtained with cumulative doses of methacholine ranging between 0.117 and $30.08 \mu \mathrm{mol}$, well below those administered to mild asthmatics and normal subjects in maximal bronchial challenge tests. Higher doses of methacholine should be included in future protocols to study the pathophysiology of maximal airway narrowing.

In conclusion, in this group of asthmatic children the slopes of MDRC could not be extrapolated from the early part of DRC. Even though it is not clear which segment 
of the slope is more important as a marker of hyperresponsiveness, this should be taken into account when planning clinical research protocols.

Acknowledgments. We thank Dr. Paul Jones and Prof. Pedro Costa for their helpful comments and criticism of the manuscript.

\section{References}

1. Abramson MJ, Sauders NA, Hensley MJ (1990) Analysis of bronchial reactivity in epidemiological studies. Thorax 45:924-929

2. Aerts JGJV, Bogaard JM, Overbeek SE, Verbraak AFM, Thio P (1994) Extrapolation of methacholine $\log$ dose-response curves with a cumulative Gaussian distribution function. Eur Respir J 7:895-900

3. Anderson SD, Du Toit JI, Rodwell LT, Jenkins CR (1994) Acute effect of sodium cromoglicate on airway narrowing induced by 4.5 percent saline aerosol: outcome before and during treatment with aerosol corticosteroids in patients with asthma. Chest 105:673-680

4. Backer V (1995) Bronchial hyperresponsiveness in children and adolescents. Danish Med Bull 42:397409

5. Barnes PJ (1990) Effect of corticosteroids on airway hyperresponsiveness. Am Rev Respir Dis 141:S70S76

6. Beach JR, Stenton SC, Connolly MJ, Walters EH, Hendrick DJ (1995) Effects of diurnal variation and prolonged refractoriness on repeated measurements of airways responsiveness to methacholine. Thorax 50:235-239

7. Boulet LP, Turcotte H, Carrier G, Boutet M, Laviolette M (1995) Increased maximal airway response to methacholine during seasonal allergic rhinitis in nonasthmatic subjects: relationships with airway wall thickness and inflammation. Eur Respir J 8:913-921

8. Chai H, Farr RS, Froehlich LA, Mathison DA, McLean JA, Rosenthal RR, Sheffer AL, Spector SL, Townley RG (1975) Standardization of bronchial inhalation challenge procedures. J Allergy Clin Immunol 56:323-327

9. Charpin D, Badier M, Orehek J (1985) Dose-response curves to inhaled carbachol in asthma and chronic bronchitis. Bull Eur Physiopathol Respir 21:417-420

10. Chinn S, Burney PGJ, Britton JR, Tattersfield AE, Higgins BG (1993) Comparison of $\mathrm{PD}_{20}$ with two alternative measures of response to histamine challenge in epidemiological studies. Eur Respir J 6:670679

11. Dehaut P, Rachiele A, Martin R, Malo JL (1983) Histamine dose-response curves in asthma: reproducibility and sensitivity of different indices to assess response. Thorax 38:516-522

12. Eiser N (1991) Specificity and sensitivity of various parameters of dose-response curves. Eur Respir J $1: 41-47$

13. Engel T, Heinig JH, Madsen O, Hansen M, Weeke ER (1989) A comparison of airway responsiveness in smokers with chronic bronchitis and in asthmatic subjects. Eur Respir J 2:929-934

14. James A, Lougheed D, Pierce-Pinto G, Ryan G, Musk B (1992) Maximal airway narrowing in a general population. Am Rev Respir Dis 148:895-899

15. Malo JL, Cartier A, Pineau L, Gagnon G, Martin RR (1985) Slope of dose-response curve to inhaled histamine and methacholine and $\mathrm{PC}_{20}$ in subjects with symptoms of airway hyperexcitability and in normal subjects. Am Rev Respir Dis 132:644-647

16. National Institutes of Health (NHLBI) (1993) Global strategy for asthma management and prevention. Publication 95, p 4659

17. O'Connor G, Sparrow D, Taylor D, Segal M, Weiss S (1987) Analysis of dose-response curves to methacholine. Am Rev Respir Dis 136:1412-1417

18. O'Connor GT, Sparrow D, Weiss ST (1995) A prospective study of methacholine airway responsiveness as a predictor of pulmonary function decline: the normative aging study. Am J Respir Crit Care Med 152:87-92 
19. Orehek J, Gayrard P, Smith AP, Charpin J (1977) Airway response to carbachol in normal and asthmatic subjects: distinction between bronchial sensitivity and reactivity. Am Rev Respir Dis 115:937-943

20. Peat J, Salome CM, Bauman A, Toelle BG, Wachinger SL, Woolcock AJ (1991) Repeatability of histamine bronchial challenge and comparability with methacholine challenge in a population of Australian schoolchildren. Am Rev Respir Dis 144:338-343

21. Peat JK, Salome CM, Berry G, Woolcock AJ (1991) Relation of dose-response slope to respiratory symptoms in a population of Australian schoolchildren. Am Rev Respir Dis 144:663-667

22. Peat JK, Salome CM, Berry G, Woolcock AJ (1992) Relation of dose-response slope to respiratory symptoms and lung function in a population study of adults living in Busselton, Western Australia. Am Rev Respir Dis 146:860-865

23. Rijken B, Schouten JP (1993) Measuring bronchial responsiveness in epidemiology. Eur Respir J 6:616-617

24. Rijcken BR, Schouten JP, Weiss ST, Meinesz AF, De Vries K, Van der Lende R (1989) The distribution of bronchial responsiveness to histamine in symptomatic and in asymptomatic subjects. Am Rev Respir Dis 140:615-623

25. Rodwell LT, Anderson SD, Seale JP (1992) Inhaled steroids modify bronchial responses to hyperosmolar saline. Eur Respir Dis 5:953-962

26. Sherrill DL, Martinez FD, Sears MR, Lebowitz MD (1993) An alternative method for comparing and describing methacholine response curves. Am J Respir Crit Care Med 148:116-122

27. Sterk PJ, Bel EH (1989) Bronchial hyperresponsiveness: the need for distinction between hypersensitivity and excessive airway narrowing. Eur Respir J 2:267-274

28. Sterk PJ, Daniel EE, Zamel N, Hargreave FE (1985) Limited bronchoconstriction to methacholine using partial flow-volume curves in nonasthmatic subjects. Am Rev Respir Dis 132:272-277

29. Sterk PJ, Fabbri LM, Quanjer P, Cockcroft H, O'Byrne PM, Anderson SD, Juniper EF, Malo JL (1993) Airway responsiveness: standardized challenge testing with pharmacological, physical and sensitizing stimuli in adults. Eur Respir Dis 6:(suppl 56) 86

30. Sterk PJ, Timmers MC, Bel EH, Dijkman JH (1988) The combined effects of histamine and methacholine on the maximal degree of airway narrowing in normal humans in vivo. Eur Respir J 1:34-40

31. Sterk PJ, Timmers MC, Dijkman JH (1986) Maximal airway narrowing in humans in vivo: histamine compared with methacholine. Am Rev Respir Dis 134:714-718

32. Trigg CJ, Jhalli N, Herdmen MJ, Cundell DR, Thomas JM, Davies RJ (1990) The daily variability of bronchial responsiveness to methacholine. Eur Respir J 3:867-871

33. van Essen Zandvliet EE, Hughes MD, Waalkens HJ, Duivermann EJ, Pocock SJ, Kerrebijn KF (1992) Effects of 22 months of treatment with inhaled corticosteroids and/or beta ${ }_{2}$ agonists on lung function, airway responsiveness and symptoms in children with asthma. Am Rev Respir Dis 146:547-554

34. Woolcock AJ, Salome CM, Yan K (1984) The shape of the dose-response curve to histamine in asthmatic and normal subjects. Am Rev Respir Dis 130:71-75

Accepted for publication: 12 December 1996 